\title{
Analyzing the fundamental properties of Raman amplification in optical fibers
}

\section{Rottwitt, Karsten; Povlsen, Jørn Hedegaard}

Published in:

Journal of Lightwave Technology

Link to article, DOI:

10.1109/JLT.2005.857776

Publication date:

2005

Document Version

Publisher's PDF, also known as Version of record

Link back to DTU Orbit

Citation (APA):

Rottwitt, K., \& Povlsen, J. H. (2005). Analyzing the fundamental properties of Raman amplification in optical fibers. Journal of Lightwave Technology, 23(11), 3597-3605. https://doi.org/10.1109/JLT.2005.857776

\section{General rights}

Copyright and moral rights for the publications made accessible in the public portal are retained by the authors and/or other copyright owners and it is a condition of accessing publications that users recognise and abide by the legal requirements associated with these rights.

- Users may download and print one copy of any publication from the public portal for the purpose of private study or research.

- You may not further distribute the material or use it for any profit-making activity or commercial gain

- You may freely distribute the URL identifying the publication in the public portal

If you believe that this document breaches copyright please contact us providing details, and we will remove access to the work immediately and investigate your claim 


\title{
Analyzing the Fundamental Properties of Raman Amplification in Optical Fibers
}

\author{
Karsten Rottwitt and Jørn H. Povlsen
}

\author{
Invited Paper
}

\begin{abstract}
The Raman response of germanosilicate fibers is presented. This includes not only the material dependence but also the relation between the spatial-mode profile of the light and the Raman response in the time and frequency domain. From the Raman-gain spectrum, information is derived related to the nonlinear refractive index due to nuclear motions and the Raman response function in the time domain. It is demonstrated that the Raman-gain coefficient may be reduced up to $60 \%$ if the signal propagates in the fundamental mode while the pump alternates between the fundamental mode and a higher order mode. A simple model shows that the time response related to the decay of phonons is significantly larger in germanate glass relative to silica glass. From the Raman gain, it is found that the contribution to the nonlinear refractive index from nuclear motions is reduced by a factor of 2 in germanate relative to silica glass.
\end{abstract}

\section{INTRODUCTION}

$\mathbf{R}$ AMAN scattering is intrinsic to silica glass. This means that Raman amplification of an optical signal is achievable in any silica-based optical fiber when the signal light propagates simultaneously with a light beam, referred to as the pump beam, at a wavelength matched to the so-called Stokes shift. This Stokes shift is characteristic to the material through which the beams propagate. In silica glass, the Stokes shift is a broad distribution but with the strongest peak at $13 \mathrm{THz}$ [1]. This corresponds to $100 \mathrm{~nm}$ when the pump is around $1450 \mathrm{~nm}$, resulting in the strongest peak in the Raman amplification when the signal is located at wavelengths around $1550 \mathrm{~nm}$.

Raman amplification has proven to be an enabling technology for implementation of high-capacity optical communication systems. This is mainly because Raman gain may be provided throughout the entire transmission fiber, ensuring that the signal-to-noise ratio is improved when compared to discrete amplification. In addition to this ability to provide distributed amplification, Raman amplification also offers the possibility to provide gain at any wavelength simply by matching the signal and pump wavelengths to the Stokes shift. Furthermore, the bandwidth of a Raman amplifier may be extended by combining multiple pump wavelengths.

Manuscript received November 23, 2004.

The authors are with the Department for Communication, Optics and Materials, Technical University of Denmark, COM•DTU, Lyngby 2800, Denmark (e-mail: kar@com.dtu.dk).

Digital Object Identifier 10.1109/JLT.2005.857776
In the late 1980s, Raman amplification was perceived as the way to overcome attenuation in optical fibers, and research on long haul transmission was carried out, demonstrating transmission through several thousand of kilometers of optical fibers using distributed Raman amplification [2]. However, with the commercialization of erbium-doped fiber amplifiers (EDFAs) through the early 1990s, work on distributed Raman amplifiers was abandoned because of its poor pump-power efficiency when compared to EDFAs. In the mid-1990s, high-power pump lasers became available, and in the years after, several system experiments demonstrated the benefits of distributed Raman amplification including repeaterless undersea experiments [3], high-capacity terrestrial [4], [5] and submarine systems transmission experiments [6], shorter span single-channel systems including $320 \mathrm{~Gb} / \mathrm{s}$ pseudolinear transmission [7], and soliton systems [8], [9].

The capability to improve the noise performance by using distributed amplification was demonstrated in distributed erbium-doped amplifiers in the early 1990s [10], [11], and more recently, using distributed Raman amplification. In both schemes, the transmission fiber is, in itself, turned into an amplifier. This is a strong benefit, but at the same time, it becomes more challenging to optimize the fiber design with respect to amplifier performance since the fiber also has to be optimized for signal transmission, i.e., with constraints to, for example, the group-velocity dispersion and the nonlinearities at the signal wavelengths.

When evaluating the performance of a distributed amplifier, not only linear noise accumulation and pulse distortion due to group velocity dispersion need to be considered, but also effects due to Rayleigh scattering, nonlinear interactions between pump and signal channels, and pump-signal crosstalk are essential. These noise sources become relevant because gain is accumulated over tens of kilometers in distributed amplifiers, and because the Raman process has a very fast response time in the order of femtoseconds [11]. As a consequence of the pump-signal crosstalk, Raman amplifiers are typically configured with the pump propagating in the opposite direction as the signal. This ensures averaging of pump fluctuation during the amplification process. However, the forward-pumped amplifier configuration, where pump and signal propagate in the same direction, offers an improved linear noise performance compared to the backward-pumped amplifier. Thus, the desire to implement forward pumping has led to the development of alterna- 
tive pumping configurations of distributed Raman amplifiers, including second-order or cascaded pumping and quiet pumps. A detailed review of Raman amplifiers and their design is found in [12]. A large effort has also been published related to modeling of Raman amplifiers assuming that both pump and signal are continuous waves [13].

In recent systems, work there has been a push towards ultrahigh bit rates in a single channel, for example, in $320 \mathrm{~Gb} / \mathrm{s}$ [7]. In these systems, the pulse duration becomes short enough that the material response needs to be described by more than just the Raman-gain coefficient. This is also valid in supercontinuum generation in optical fibers, typically with a modified group-velocity-dispersion profile [14]. In addition, optimized ultrahigh-capacity systems also require the use of so-called engineered spans, where the fiber design is pushed toward its limits to minimize nonlinear penalties, while at the same time ensuring optimum dispersion compensation [15]. These efforts has motivated this work. Here, we review fundamental relations between the fiber design, and its impact on the Raman response and the intensity-dependent refractive index.

In Section II of this work, we review fundamental aspects of the Raman response in fibers, in the frequency as well as in the time domain. This is related to predictions of the Raman gain, i.e., the imaginary part of the frequency response of the Raman gain, Section III. In Section IV, we evaluate the Raman response in different fibers based on the basic materials being the main constituents of fibers. In Section IV-A, the prediction of Raman-gain coefficients in different fiber types is highlighted. In future new advanced fiber designs, the cutoff for higher order modes may be pushed towards much higher wavelengths. Thus, the pump may be multimode while the signal is single-mode or multimode. The impact of this is described in Section IV-B. When propagating short pulses at sufficient power levels, the response time of the Raman scattering needs to be accounted for. This becomes even more important as the bit rate within a single channel is pushed beyond hundreds of gigabits per second and with the increasing interest in supercontinuum generation. Section IV-C describes the Raman response time in various fiber types. In Section IV-D, the contribution to the nonlinear refractive index due to Raman scattering is discussed. Finally, Section V concludes the paper.

\section{THEORY: THE INDUCED POLARIZATION}

In the Raman process, light interacts with the glass network, which on a microscopic scale is rotating and vibrating at room temperature. As a consequence of this, the electrical field, i.e., the pump and signal, induces a macroscopic polarization. At sufficiently high power levels, the induced polarization relates nonlinearly to the electrical field. In the time domain, the electrical field vector $\mathbf{E}$ is, in general, governed by the wave equation

$$
\nabla \times \nabla \times \mathbf{E}+\frac{1}{c^{2}} \frac{\partial^{2}}{\partial t^{2}} \mathbf{E}=-\mu_{0} \frac{\partial^{2}}{\partial t^{2}} \mathbf{P}
$$

where $\mathbf{P}$ is the macroscopic nonlinear induced polarization vector, $c$ is the speed of light, and $\mu_{0}$ is the magnetic permeability. In glass materials, the induced polarization has a linear and a nonlinear part. The linear part describes the refractive index, whereas Raman scattering is described through the nonlinear third order induced polarization. In general, both the electric field and the macroscopic polarization depend upon both position and time. However, in the following, we assume that the macroscopic polarization is local, and thus, the induced thirdorder polarization is related to the response $\mathbf{T}^{(3)}\left(t_{0}, t_{1}, t_{2}, t_{3}\right)$ through [16]

$$
\begin{array}{r}
\mathbf{P}^{(3)}\left(t_{0}\right)=\varepsilon_{0} \int_{-\infty}^{\infty} \int_{-\infty}^{\infty} \int_{-\infty}^{\infty} \mathbf{T}^{(3)}\left(t_{0}, t_{1}, t_{2}, t_{3}\right) \\
\times \mathbf{E}\left(t_{1}\right) \mathbf{E}\left(t_{2}\right) \mathbf{E}\left(t_{3}\right) \mathrm{d} t_{1} \mathrm{~d} t_{2} \mathrm{~d} t_{3}
\end{array}
$$

where $\mathbf{T}^{(3)}\left(t_{0}, t_{1}, t_{2}, t_{3}\right)$ is a tensor of rank 4 , which only depends on the differences among the time arguments [16]. It is noted that the response $\mathbf{T}^{(3)}\left(t_{0}, t_{1}, t_{2}, t_{3}\right)$ vanishes for either $t_{1}<t_{0}, t_{2}<t_{0}$, or $t_{3}<t_{0}$ due to causality.

In the Raman-scattering process, the response function consists of an electronic and a nuclear response. The latter originates from vibrations and rotations of molecules [17]. Assuming that the material response may be expressed through the electronic response $\sigma$ and the nuclear response $h$ as

$$
\begin{aligned}
\mathbf{T}^{(3)}=\sigma \delta\left(t_{0}-t_{1}\right) \delta( & \left.t_{1}-t_{2}\right) \delta\left(t_{2}-t_{3}\right) \\
& +\delta\left(t_{0}-t_{1}\right) \mathbf{h}\left(t_{1}-t_{2}\right) \delta\left(t_{2}-t_{3}\right)
\end{aligned}
$$

the $i$ th component of the macroscopic polarization is from (2)

$$
\begin{aligned}
P_{i}^{(3)}\left(t_{0}\right)= & \sigma_{i j k l} \varepsilon_{0} E_{j}\left(t_{0}\right) E_{k}\left(t_{0}\right) E_{l}\left(t_{0}\right) \\
& +E_{j}\left(t_{0}\right) \varepsilon_{0} \int_{-\infty}^{\infty} h_{i j k l}\left(t_{0}-t_{2}\right) E_{k}\left(t_{2}\right) E_{l}\left(t_{2}\right) \mathrm{d} t_{2}
\end{aligned}
$$

where $(i, j, k, l)$ represent Cartesian coordinates. In (4) and in the following, a summation is implicitly assumed over all indices $j, k$, and $l$ on the right-hand side according to the Einstein tensor notation.

For glass, and more generally for isotropic materials, the electronic response tensor reduces to a scalar and the induced polarization $\mathbf{P}_{\mathrm{e}}^{(3)}$ due to the electronic response equals

$$
\mathbf{P}_{\mathrm{e}}^{(3)}=\sigma \varepsilon_{0}(\mathbf{E}(t) \cdot \mathbf{E}(t)) \mathbf{E}(t)
$$

whereas the nuclear response may be approximated by the sum of only two independent contributions [16] in the form

$$
h_{i j k l}(t)=a(t) \delta_{i j} \delta_{k l}+\frac{1}{2} b(t)\left[\delta_{i l} \delta_{j k}+\delta_{i k} \delta_{j l}\right]
$$

where $\delta_{i j}$ is the Kronecker delta function, which is equal to 1 when the indices are equal, and is equal to 0 otherwise. This equation also shows that the only nonzero tensor elements are the elements where indices appear in pairs and that $h$ is invariant to the interchange of either its first or last two indices. In addition, $h$ is invariant to an exchange of is first pair of indices with its last. Finally, these rules imply that $h_{i i i i}=$ $h_{i i j j}+h_{i j j i}+h_{i j i j}$ and $h_{i j i j}=h_{i j j i}$. 
Inserting this response into (4), the induced polarization due to the nuclear response $\mathbf{P}_{\mathrm{R}}^{(3)}$ in glass materials is then

$$
\begin{aligned}
\mathbf{P}_{\mathrm{R}}^{(3)}(t)=\varepsilon_{0} \mathbf{E}(t) & \int_{-\infty}^{\infty} a(t-s) E^{2}(s) \mathrm{d} s \\
& +\varepsilon_{0} \int_{-\infty}^{\infty} b(t-s)[\mathbf{E}(t) \cdot \mathbf{E}(s)] \mathbf{E}(s) \mathrm{d} s .
\end{aligned}
$$

In the frequency domain, the response, i.e., the susceptibility tensor $\chi^{(3)}$ is found from a simple Fourier transform of the response function $\mathbf{T}$. Assuming that the electronic response is a delta function, the electronic contribution to the susceptibility is a constant for all frequencies, whereas the Fourier transform of the nuclear response $\mathbf{h}(t)$ in (6) is

$$
H_{i j k l}(\Omega)=A(\Omega) \delta_{i j} \delta_{k l}+\frac{1}{2} B(\Omega)\left[\delta_{i l} \delta_{j k}+\delta_{i k} \delta_{j l}\right]
$$

where $\Omega$ is the frequency difference between the pump and the signal. This is an important result. It implies that in the case when the electric field consists of monochromatic waves, the induced polarization in the frequency domain reduces to simple products of the frequency response corresponding to the involved frequencies times the electric-field amplitudes.

\section{Fiber WAVE EQuation}

In the following sections, we focus solely on the propagation of light interacting with phonons in the glass.

In the simplest Raman amplifier, the electric field is a superposition of two quasi- monochromatic waves: one at the pump frequency and one at the frequency of the signal wave. In the following, we use indexes $s$ and $p$ to distinguish the signal and pump, respectively. In the amplifying process, energy is transferred from the pump to the signal, and at the same time, energy is given to the fiber in terms of vibrational quanta.

When restricting the description to the most common fiber types, i.e., weakly guiding fibers, the electric field may be separated into a transverse part $R^{i}(r), i=\mathrm{s}, \mathrm{p}$, and $r=(x, y)$, and an amplitude function depending upon $z: E^{i}(z), i=\mathrm{s}$, p. The total electric field is then the sum of the electric field at the pump frequency $\omega_{\mathrm{p}}$ and the signal frequency $\omega_{\mathrm{s}}$

$$
\begin{aligned}
\mathbf{E}=\hat{\mathbf{e}}_{\mathrm{p}} E^{\mathrm{p}}(z) R^{\mathrm{p}}(r) \exp \left\{i\left(\beta_{\mathrm{p}} z-\omega_{\mathrm{p}} t\right)\right\} \\
+\hat{\mathbf{e}}_{\mathrm{s}} E^{\mathrm{s}}(z) R^{\mathrm{s}}(r) \exp \left\{i\left(\beta_{\mathrm{s}} z-\omega_{\mathrm{s}} t\right)\right\}+\text { c.c. }
\end{aligned}
$$

where $\hat{\mathbf{e}}_{i}, i=\mathrm{s}, \mathrm{p}$, is a unit polarization vector, and $\beta_{i}, i=\mathrm{s}, \mathrm{p}$, is the propagation constant, as determined from the waveguide eigenvalue problem $\left[\nabla_{\perp}^{2}+\left(\omega_{i}^{2} n_{i}^{2} / c^{2}\right)\right] R^{i}=\beta_{i}^{2} R^{i}$, where $n_{i}$ is the refractive index at the pump or signal wavelength.

Adopting the weakly guiding approximation, i.e., $\boldsymbol{\nabla} \cdot \mathbf{E}=0$, the wave equation in the frequency domain becomes

$$
-\nabla^{2} \mathbf{E}=\frac{n^{2}}{c^{2}} \omega_{\mathrm{s}}^{2} \mathbf{E}+\frac{\omega_{\mathrm{s}}^{2}}{\varepsilon_{0} c^{2}} \mathbf{P}
$$

Considering only the electrical field at the frequency of the signal $\mathbf{E}^{\mathrm{S}}$ and assuming that the state of polarization for the pump and the signal fields does not change during propagation, and neglecting $\partial^{2} \mathbf{E}^{\mathrm{s}} / \partial z^{2}$, we obtain a wave equation for the $i$ th component of the electrical signal vector

$$
\begin{aligned}
\int_{A} \frac{\partial E_{i}^{\mathrm{s}}}{\partial z}\left|R^{\mathrm{s}}\right|^{2} \mathrm{~d} A=\int_{A} \frac{\omega_{\mathrm{s}}^{2}}{2 c^{2} \beta_{\mathrm{s}}} & i\left|R^{\mathrm{s}}\right|^{2}\left|R^{\mathrm{p}}\right|^{2} 6 \\
& \times \sum_{j k l} \chi_{i j k l}^{(3)} E_{j}^{\mathrm{p}}\left(E_{k}^{\mathrm{p}}\right)^{*} E_{l}^{\mathrm{s}} \mathrm{d} A
\end{aligned}
$$

where the integration is over the entire fiber cross section. From this equation, the Raman gain is described through the imaginary part of the third-order susceptibility whereas the real part relates to and induced change in the refractive index. When pump and signal are polarized along the same axis, $\chi_{i i i i}^{(3)}$ is given by the sum: $A(\Omega)+B(\Omega)$ in (8), whereas when the pump and signal waves are polarized along orthogonal axes, $\chi_{i j j i}^{(3)}=B(\Omega) / 2$. Using polarization-maintaining fibers, it is possible to measure the Raman gain in the two cases. In [18] and [19], such a measurement shows that the cross coupling is ten times lesser than the parallel coupling and, in addition, that the cross coupling peaks at a much lower frequency difference between signal and pump when compared to parallel pumping.

Using the power $P_{\mathrm{s}}=2 \varepsilon_{0} n_{\mathrm{s}} c \int_{A}\left|E^{\mathrm{s}} R^{\mathrm{s}}\right|^{2} \mathrm{~d} A$, where $n_{\mathrm{s}}$ is the refractive index at the signal frequency and the integral is over the cross section, and introducing the effective area as the overlap between the pump and signal through

$$
A_{\mathrm{eff}}^{\mathrm{ps}}=\frac{\int_{A}\left|R_{\mathrm{p}}\right|^{2} \mathrm{~d} A \int_{A}\left|R_{\mathrm{s}}\right|^{2} \mathrm{~d} A}{\int_{A}\left|R_{\mathrm{p}}\right|^{2}\left|R_{\mathrm{s}}\right|^{2} \mathrm{~d} A}
$$

the rate equation for the signal power is

$$
\frac{\mathrm{d} P_{\mathrm{s}}}{\mathrm{d} z}=g_{r} P_{\mathrm{p}} P_{\mathrm{s}}
$$

where $g_{r}$ is the gain coefficient of the fiber. In the case when the pump and signal are plane polarized along the same axis, $g_{r}$ is given through

$$
g_{r}=-\frac{3 \omega_{\mathrm{s}}}{\varepsilon_{0} c^{2} n_{\mathrm{p}} n_{\mathrm{s}}} \frac{\operatorname{Im}[A(\Omega)+B(\Omega)]}{A_{\mathrm{eff}}^{\mathrm{ps}}}
$$

where it is assumed that the susceptibilities are constant across the entire fiber cross section, and $\beta_{\mathrm{s}}$ is approximated by $\omega_{\mathrm{s}} \bar{n} / c$ where $\bar{n}$ is the effective refractive index of the signal mode, i.e., $\beta_{\mathrm{s}}$ is proportional to $\omega_{\mathrm{s}}$.

If the pump is unpolarized, as in many practical cases, the Raman gain may approximately be found from (14) by dividing by 2 , under the assumption of a vanishing contribution from $B(\Omega)$ and an equal pump-power distribution among the two polarizations.

\section{RAMAN-SCATTERING IN FIBERS}

One of the most important applications of stimulated Raman scattering is in optical amplifiers. Since Raman scattering is 
intrinsic to silica-based fibers, optical amplification in silicabased fibers is simply achieved by propagating a pump beam together with the signal beam. The frequency spacing between the pump and signal is determined by the Stokes shift, which, in silica, gives the maximum gain when the pump frequency is $13 \mathrm{THz}$ higher than the signal frequency. Since the amplification happens in the transmission fiber itself, and over long distances, the amplification is distributed, which allows for improved linear noise accumulation.

One of the first experiments that demonstrated the improved noise performance of distributed amplification was in an undersea-system experiment, where remotely pumped inline EDFAs were pumped at $1480 \mathrm{~nm}$ [20]. In this experiment, $145 \mathrm{~mW}$ of pump power was launched into a $66-\mathrm{km}$-long dispersion-shifted fiber (DSF) at the receiver side. This gave a Raman gain of $5.3 \mathrm{~dB}$ for the signal at $1558 \mathrm{~nm}$. The remaining pump power launched into the remote EDFA was $3.8 \mathrm{~mW}$, giving rise to a 15.4-dB gain in the EDFA. The power budget was improved by $11.0 \mathrm{~dB}$ due to the remote EDFA and the Raman gain. This demonstrated the potential application of distributed Raman amplification as a means to upgrade existing systems.

Equation (13) provides the Raman gain in the absence of intrinsic fiber attenuation. However, taking the attenuation into account, the signal gain, i.e. the signal output power relative to the signal input power is $G=\exp \left(g_{r} L_{\text {eff }} P_{\mathrm{p}}^{0}\right)$ $\exp \left(-\alpha_{\mathrm{s}} L\right)$, where $L_{\mathrm{eff}}$ is the effective length $\left[L_{\mathrm{eff}}=(1-\right.$ $\left.\left.\exp \left(-\alpha_{\mathrm{p}} L\right) / \alpha_{\mathrm{p}}\right)\right], \alpha_{\mathrm{p}}$ is the fiber attenuation at the pump wavelength, $L$ is the physical length, $P_{\mathrm{p}}^{0}$ is the pump power at launch, and $\alpha_{\mathrm{s}}$ is the fiber attenuation at the signal wavelength. The first term in the gain is often referred to as the ON-OFF Raman gain $G_{\mathrm{R}}\left[G_{\mathrm{R}}=\exp \left(g_{r} L_{\mathrm{eff}} P_{\mathrm{p}}^{0}\right)\right]$, and for a typical highcapacity transmission fiber, the ON-OFF Raman approximates $60 \mathrm{~dB} / \mathrm{W}$ of pump power, assuming a physical length exceeding the effective length $\left(L \gg L_{\text {eff }}\right)$.

The noise figure, i.e., the degradation of the signal-tonoise ratio when the signal has passed through the amplifier, is slightly more complicated to evaluate. The noise power $P_{\mathrm{n}}$ is [21]

$$
P_{\mathrm{n}}=\hbar \omega_{\mathrm{s}} B_{0} \eta_{\mathrm{T}} g_{\mathrm{R}} \int_{z=0}^{L} \frac{P_{\mathrm{p}}(z)}{G(z)} \mathrm{d} z
$$

where $\hbar \omega_{\mathrm{s}} B_{0}$ is the power of a photon within the bandwidth $B_{0}$, and $\eta_{\mathrm{T}}$ is the thermal equilibrium phonon number [22]; at room temperature, $\eta_{\mathrm{T}} \approx 1.14$. From the noise power, the noise figure $F$ is calculated from

$$
F=\frac{\left(\frac{2 P_{\mathrm{n}}}{\left(\hbar \omega_{\mathrm{s}} B_{0}\right)}+1\right)}{G} .
$$

For a backward-pumped amplifier, where the gain exactly compensates the intrinsic loss, i.e., $G_{\mathrm{R}}=\exp \left(\alpha_{s} L\right)$, the noise figure equals

$$
F=1+2 \frac{\eta_{\mathrm{T}} \alpha_{\mathrm{s}}}{g_{\mathrm{R}} P_{\mathrm{p}}^{0}}\left(G_{\mathrm{R}}-1\right)
$$

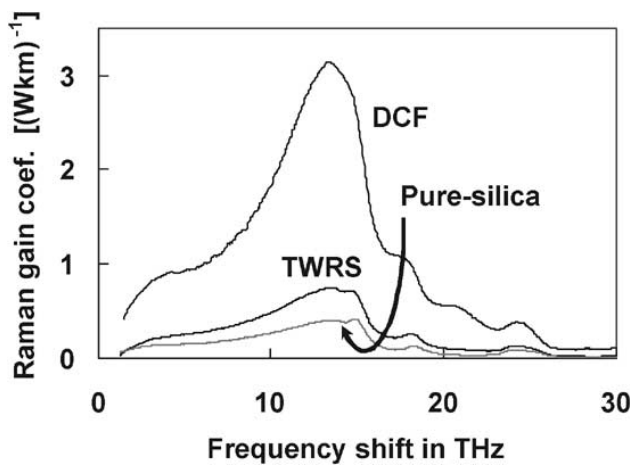

Fig. 1. Raman-gain coefficient versus frequency shift between pump and signal for different fiber types. In the dispersion-shifted fiber (DSF), the germanium content is higher and the effective area is less than that in the high-capacity transmission fiber, the TrueWave Reduced Slope (TWRS) fiber supplied by OFS. The pure-silica fiber has no germanium in the core.

In optimized Raman amplifiers, this noise figure is typically less than the signal attenuation in a transmission fiber, which explains the improved noise performance [23]. It is noted that the noise figure of a passive fiber equals the signal attenuation of the fiber.

Fig. 1 displays the Raman-gain coefficient for different fiber types. All are measured using unpolarized light and a pump of $1453 \mathrm{~nm}$. The pure silica fiber (Pure-silica), which has a fiber core of silica and a cladding with a lower refractive index, displays the lowest gain coefficient, with a peak value of approximately $0.5(\mathrm{~W} \cdot \mathrm{km})^{-1}$. The dispersion-compensating fiber (DCF), which has a silica cladding and a core of germaniumdoped silica glass, displays the largest Raman-gain coefficient, with a peak value of approximately $3(\mathrm{~W} \cdot \mathrm{km})^{-1}$. The higher gain coefficient, when compared to the silica core fiber, is caused by the germanium content and the effective core area [24]. In typical high-capacity transmission fibers with a lower germanium content relative to the DCF fiber, exemplified by a TrueWave Reduced Slope fiber (TWRS), as seen in Fig. 1, the gain coefficient is in the order of $1(\mathrm{~W} \cdot \mathrm{km})^{-1}$ at its peak, and $A_{\text {eff }}^{\mathrm{ps}} \approx 75 \times 10^{-12} \mathrm{~m}^{2}$.

In the recent decade, there has been a significant research effort into the design of Raman amplifiers, which has led to significant conquests within the application of Raman amplifiers [25]. In the following, we focus on the Raman-gain coefficient and the implications of Raman scattering in optical fibers.

\section{A. Raman-Gain Coefficient in Optical Fibers}

The Raman-gain coefficient is one of the essential parameters in the design of fibers for Raman amplifiers. Thus, it is critical to be able to predict the Raman-gain coefficient. The composite spectrum of any germanosilicate spectrum, with moderate fractional germanium concentration (less than 50\%) [24], may be found if the germanium concentration is known together with either the refractive index profile or the effective area [22]. This is done on the basis of a material spectrum related to silica-oxygen-silica ( $\mathrm{Si}-\mathrm{O}-\mathrm{Si}$ ) bonds and another spectrum related to germanium-oxygen-silica ( $\mathrm{Si}-\mathrm{O}-\mathrm{Ge})$ bonds [24]. These spectra are illustrated in Fig. 2 together with the spectrum for germanate glass, $\mathrm{GeO}_{2}$. 


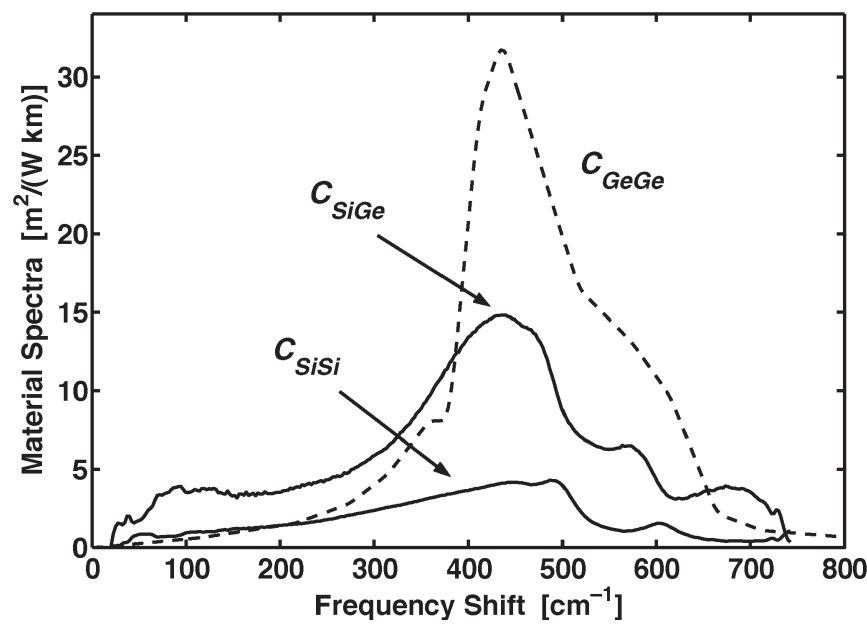

Fig. 2. Material spectra for the Raman-scattering process in fibers. The spectra for $\mathrm{Si}-\mathrm{O}-\mathrm{Si}, C_{\mathrm{SiSi}}$ and for $\mathrm{Si}-\mathrm{O}-\mathrm{Ge}, C_{\mathrm{SiGe}}$ are found based on measured data of optical fibers, whereas the spectrum for $\mathrm{GeO}_{2}, C_{\mathrm{GeGe}}$, is adapted from [26] applying a scaling of 7.7 to the peak of the $\mathrm{Si}-\mathrm{Si}$ spectrum [27], as explained in the text.

It is important to note that there are significant differences between the spectrum related to germanium-oxygen-silica bonds and the spectrum related to germanate glass.

The figure displays the material parameters related to the Raman-gain coefficient. However, the spectrum related to germanate glass is based on measurements in bulk samples. In bulk samples, the Raman scattering is characterized by its scattering cross section. Germanate glass has been reported to have a Raman cross section that is 9.2 times higher than that of silicate glass. However, in translating between cross sections and gain coefficients, there is a scaling with the inverse of the square of the refractive index of the samples. Thus, using a refractive index of silica of $n_{\mathrm{SiO}_{2}}=1.46$, and a refractive index of germanate of $n_{\mathrm{GeO}_{2}}=1.6$, the gain coefficient of $\mathrm{GeO}_{2}$ is expected to be 7.7 times higher than that of $\mathrm{SiO}_{2}$.

From the spectra in Fig. 2, the composite spectrum is found according to

$$
\begin{aligned}
g_{r}=C_{\mathrm{SiSi}} \int_{0}^{\infty}(1- & 2 x(r)) I^{\mathrm{p}}(r) I^{\mathrm{s}}(r) r \mathrm{~d} r \\
& +C_{\mathrm{SiGe}} \int_{0}^{\infty}(2 x(r)) I^{\mathrm{p}}(r) I^{\mathrm{s}}(r) r \mathrm{~d} r
\end{aligned}
$$

where $x(r)$ is the fractional molar germanium concentration, and $1-2 x(r)$ and $2 x(r)$ are the fractional molar concentration of $\mathrm{Si}-\mathrm{O}-\mathrm{Si}$ and $\mathrm{Si}-\mathrm{O}-\mathrm{Ge}$ bonds, respectively. $I^{\mathrm{p}}(r)$ and $I^{\mathrm{s}}(r)$ are the radial intensity distributions for the pump and signal, respectively, normalized such that $\int_{0}^{\infty} I^{i}(r) r \mathrm{~d} r=1, i=\mathrm{s}, \mathrm{p}$. The two material spectra $C_{\mathrm{SiSi}}$ and $C_{\mathrm{SiGe}}$ are displayed in Fig. 2.

The spectra in Fig. 2 are the spectra valid for the case when the pump is unpolarized. To obtain the spectra if both pump and signal are plane polarized along the same axis, the data in the figure are multiplied by a factor of 2 . The gain coefficients in Fig. 2 are based on measured data for a pump wavelength

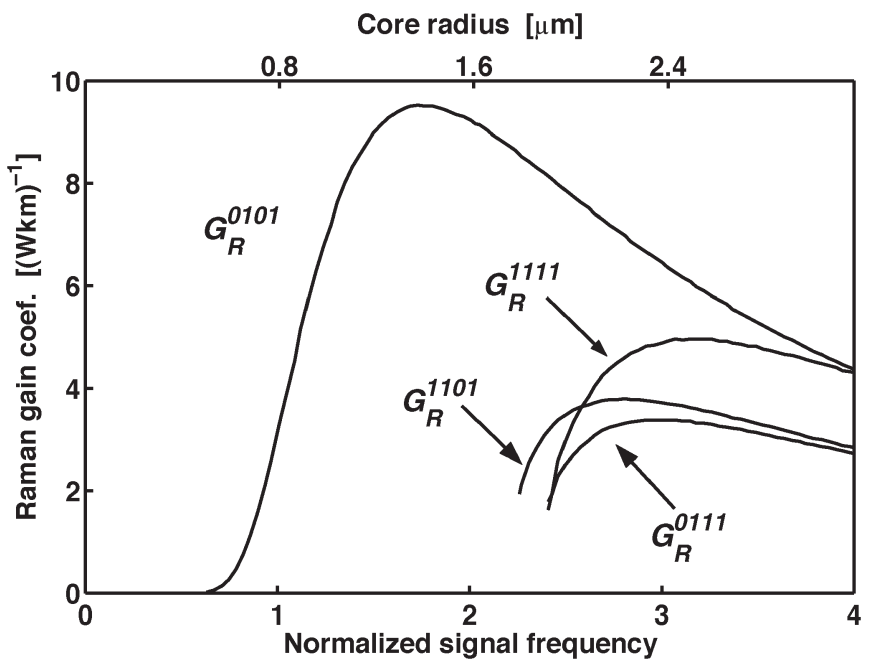

Fig. 3. Calculated Raman-gain coefficients versus the normalized frequency at the signal wavelength. Each curve is labeled according to the mode of the pump and signal, i.e., $G_{\mathrm{R}}^{1101}$ is the gain coefficient corresponding to the pump being in the $\mathrm{LP}_{11}$ mode and the signal in the $\mathrm{LP}_{01}$ mode. The relatively large gain coefficients are due to the high index contrast between core and cladding, i.e., a large content of germanium in the core of the fiber.

at $\lambda_{\mathrm{p}}=1455 \mathrm{~nm}$. Scaling to another pump wavelength $\lambda_{\mathrm{p}}^{\prime}$ is done by multiplying by $\lambda_{\mathrm{p}} / \lambda_{\mathrm{p}}^{\prime}$.

\section{B. Raman Coupling Between Spatial Modes}

In some transmission fibers, the fiber design is optimized with respect to, for example, dispersion properties or effective area. An example of such a system is an engineered span system [15]. In this process, the cutoff for the first higher order mode of the fiber may be pushed close to the wavelength of the pump. In such a case, the pump may become multimode, whereas the signal may still be single mode. To the first order, this leads to a reduced Raman efficiency; but even more critical, it may lead to an additional noise contribution since some of the pump power may alternate randomly between being in the fundamental mode and in a higher order mode.

The relation between the Raman gain and the spatial overlap of the pump and signal mode was described in Section III. However, in this section, we evaluate the Raman-gain coefficient when the pump and signal appear in different spatial modes. A careful evaluation of potential induced noise is considered outside the scope of this paper.

In Fig. 3, the Raman-gain coefficient, for a frequency shift of $13 \mathrm{THz}$ between pump and signal, is illustrated for the case where the pump and signal are in the same polarization. In the figure, the radius of the fiber core $a$ in a step index fiber with an extremely high germanium concentration $20 \mathrm{~mol} \%$ is varied to describe the single-mode and multimode operations. A change in the core radius is analogous to a change in the normalized frequency $V$ defined through $V=k a \sqrt{n_{0}^{2}-n_{1}^{2}}$, where $k$ is the wavenumber of the signal, and $n_{0}$ and $n_{1}$ are the refractive indexes of the core and cladding, respectively. A pump of $1450 \mathrm{~nm}$ and a signal at $1550 \mathrm{~nm}$ is assumed. When $V$ exceeds 2.405 , the fiber becomes multimode. For a core diameter below $3.6 \mu \mathrm{m}$, the fiber is single mode for both pump and signal, $G_{\mathrm{R}}^{0101}$. Note that even in this case, the fiber still supports 
two modes of orthogonal polarizations, leading to polarizationdependent gain [28].

In the single-mode region for both pump and signal, the maximum Raman-gain coefficient is found for a core diameter close to $2.8 \mu \mathrm{m}$. This is the geometry that provides the maximum overlap of both the pump and signal modes and their overlap to the core.

When the diameter of the core is between 3.6 and $3.8 \mu \mathrm{m}$, the pump becomes multimode while the signal is still single mode. As a consequence, the Raman-gain coefficient may now assume two different values depending on whether the pump is in the fundamental mode, $G_{\mathrm{R}}^{0101}$, or in the higher order mode, $G_{\mathrm{R}}^{1101}$. In addition to the reduced Raman efficiency of the amplifier (gain per unit pump power), the variation in Ramangain coefficient may translate into a noise contribution, if, for example, the power in one mode at some random distance within the span, or at some random ratio, transfers to the other mode [29].

Finally, when the diameter of the core exceeds $3.8 \mu \mathrm{m}$, both the pump and signal become multimode. Thus, the Ramangain coefficient may now assume several values because of the degeneracy of pump and signal modes, also including the degeneracy in the angular coordinate of a given cross section of the fiber [30]. Assuming that the pump and signal are in the same polarization state and also the same trigonometric mode, the data in Fig. 3 are found for the Raman-gain coefficients as a function of the core radius of the fiber.

If the core diameter exceeds $5.7 \mu \mathrm{m}$, even more modes are guided, and the description gets further complicated.

This shows that there is a strong dependence in the Ramangain coefficient due to the mode profile of the pump and signal. In the illustrated example, this may potentially lead to a $60 \%$ reduction in the Raman-gain coefficient if the signal is single mode, but the pump alternates between the fundamental mode and the first higher order mode.

\section{Response Time}

The response time of the Raman process is related to nuclear rotations and vibrations, and hence, longer than the electronic response time. In [31], the Raman response time of fused Silica is evaluated to be less than 100 fs. For most applications, this appears instantaneous, and especially in relation to the application as Raman amplifiers in optical communications systems, the Raman-gain coefficient provides sufficient information. This is especially true because the propagation direction of the pump is typically chosen to be opposite to that of the signal to mitigate unwanted crosstalk between the pump and the signal. In the case when pump and signal propagate in the same direction, fluctuations in the pump translate to fluctuations in the signal; this is enhanced due to the fast response time from the Raman process.

When describing pulse propagation, the response time of the Raman-scattering process is becoming an important parameter. This is because the pulse duration of a single bit in a single channel as well as in a multiple wavelength channel communications system is pushed close to a picosecond or below. In both cases, the response time needs to be included

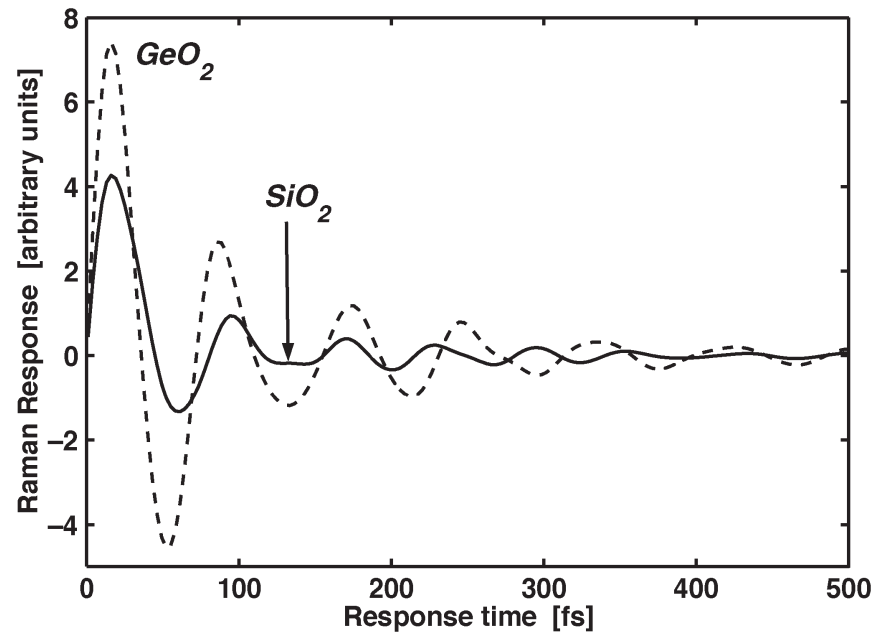

Fig. 4. Raman response function. The response is normalized according to $\int h(t) \mathrm{d} t=1$.

even in a system that is not applying Raman amplification. For the system with multiple wavelengths, or Raman amplified, the propagation equation is further complicated because of the mutual interaction among various channels, e.g., cross-phase modulation or interactions between the signal and the pump beams for the Raman amplifier [32], [33]. This will not be considered in this paper.

A topic that has received much attention lately is supercontinuum generation of light [14]. Supercontinuum may be achieved by launching short pulses into an optical fiber with enhanced nonlinear effects obtained by reducing the effective area and a group-velocity dispersion tailored to favor generation of light within a broad spectral range. In this case, the response function is critical for predicting the supercontinuum generation in specialty fibers including microstructured photonic crystal fibers.

The Raman response function varies from material to material, similar to the Raman-gain coefficient. Hence, it is difficult to obtain accurate knowledge of the response function from fiber to fiber. However, the response function of the Ramanscattering process $[h(t)$ in (6)] may be directly evaluated through the Fourier transform of the Raman susceptibility of $H(\Omega)$, e.g., as discussed in Section II.

Fig. 4 displays both the response from $\mathrm{SiO}_{2}$ and the response from $\mathrm{GeO}_{2}$. From the figure, it is clear that the response time for germanium is larger than silicium, which we attribute to the larger atomic weight of germanium relative to silicium. However, both of the materials have a response time close to 100 fs, with a difference less than $10 \%$.

In a simple picture, the response time may be fitted to a single damped harmonic oscillator with two time constants $\tau_{\nu}$, which is related to the frequency of the "phonon," and $\tau_{\mathrm{s}}$, which is related to the attenuation of the network of vibrating atoms, i.e.,

$$
h(t)=a \exp \left(\frac{-t}{\tau_{\mathrm{s}}}\right) \sin \left(\frac{t}{\tau_{\nu}}\right) .
$$

For the two curves in Fig. 4, we find the best fit to (19) with the time constants listed in Table I. 
TABLE I

Time Constants of THE DAMPED HARMONiC OsCillator RELATED TO THE RAMAN RESPONSE FUNCTION. THE LAST COLUMN Noted With THE* IS AdOPTED FROM [34]

\begin{tabular}{|l|c|c|c|}
\hline Material & $\mathrm{GeO}_{2}$ & $\mathrm{SiO}_{2}$ & $\mathrm{SiO}_{2}{ }^{*}$ \\
\hline \hline$\tau_{\nu}[\mathrm{fs}]$ & 12 & 13 & 12 \\
\hline$\tau_{s}[\mathrm{fs}]$ & 83 & 39 & 32 \\
\hline
\end{tabular}

From the table, there is no noticeable difference between the time constant related to the phonon frequency whereas there is a significant difference in the time constant related to the damping of the phonons.

A more comprehensive modeling of the response function requires more insight into the possible rotational and vibrational modes of the glass network. Such a modeling, provided in [35] and [36], has shown that it is necessary to combine an ensemble of damped oscillators to get an accurate fit to the response function. In this intermediate broadening model, which involves neither homogeneously nor inhomogeneously broadened oscillators, the Raman response is modeled as an ensemble of 13 damped harmonic oscillators with a Gaussian distribution of the mean frequencies. As a result, the gain spectrum is a convolution of a Gaussian and a Lorentz form, and the response function has a perfect match.

\section{Relation Between Raman Gain and Intensity-Dependent Refractive Index}

The propagation equation (11) shows that the real part of the susceptibility is equivalent to an induced change in the refractive index. In the case of Raman scattering, this implies that the nuclear ro-vibrations causes a change in the refractive index of the signal induced by the pump. If the frequency of the pump equals the frequency of the signal, the effect is equivalent to self-induced change in the refractive index or self-phase modulation. In the following, we discuss this effect.

First, we consider the case when the frequency of the pump and signal is identical. In this case, the transverse dependence of the pump now equals that of the signal: $R^{\mathrm{p}}=R^{\mathrm{s}}$. From (11), the electric field is then governed by

$$
\frac{\partial E_{i}^{\mathrm{s}}}{\partial z}=\frac{\omega_{\mathrm{s}}^{2}}{2 c^{2} \beta_{\mathrm{s}}} i\left|R^{\mathrm{s}}\right|^{2} 6 \sum_{j k l} \operatorname{Re}\left[\chi_{\mathrm{ijkl}}^{(3)}\right] \mathrm{E}_{\mathrm{j}}^{\mathrm{s}}\left(\mathrm{E}_{\mathrm{k}}^{\mathrm{s}}\right)^{*} \mathrm{E}_{\mathrm{l}}^{\mathrm{s}} .
$$

Assuming, for simplicity, that the signal is linearly polarized, this leads to a nonlinear refractive index $n_{2}$ given by

$$
n_{2}=\frac{3}{4 n_{\mathrm{s}}^{2} c \varepsilon_{0}} \frac{\operatorname{Re}\left[\chi_{\mathrm{iiii}}^{(3)}\right]}{\mathrm{A}_{\mathrm{eff}}}
$$

where $A_{\text {eff }}$ is identical to $A_{\text {eff }}^{\mathrm{ps}}$ in (14), with $R^{\mathrm{p}}=R^{\mathrm{s}}$.

From (6), the response is a sum of an electronic contribution $\sigma$, which is instantaneous, and a nuclear contribution $h(t)$ with a slower response time, discussed in Section IV-C. In the frequency domain, the contribution to the self-induced refractive index is found at $\Omega=0$.

Comparing $n_{2}$ in (2) and $g_{r}$ in (14), it is seen that $n_{2}$ may be found from $g_{r}$ by interchanging the imaginary part of $\chi^{(3)}$ with

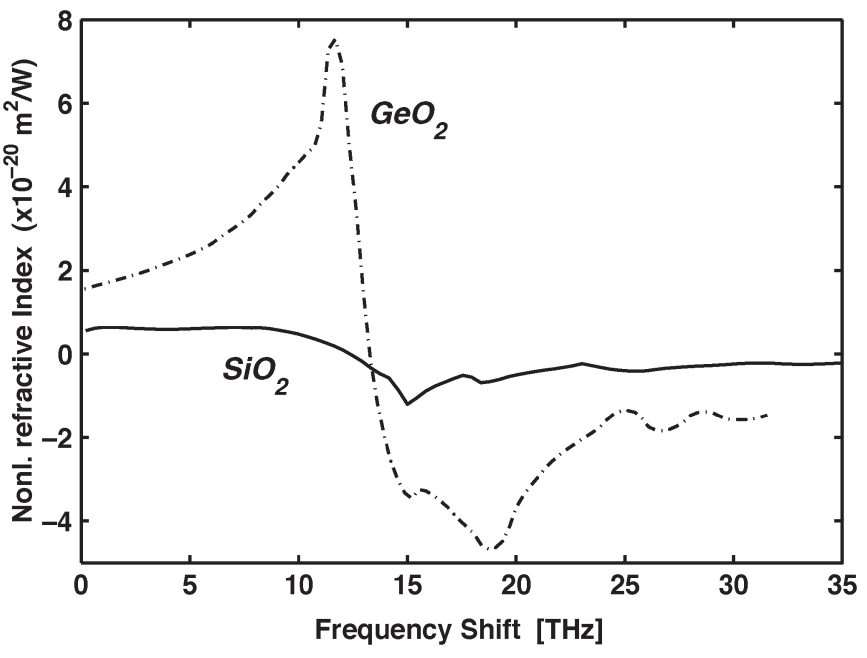

Fig. 5. Raman-induced contribution to the nonlinear refractive index. The contribution from $\mathrm{GeO}_{2}$ is 3.5 times higher than that from $\mathrm{SiO}_{2}$. In the figure, contributions from the orthogonal Raman scattering $B(\Omega)$ is neglected. The spectrum used to generate the $\mathrm{SiO}_{2}$ contribution is from [31]. The curve for $\mathrm{GeO}_{2}$ is calculated assuming a gain coefficient 7.7 times that of $\mathrm{SiO}_{2}$ [27] and a spectral shape adopted from [26].

the real part and multiplying by $c / 4 \omega$. Thus, the Raman contribution to the self-induced refractive index may be obtained from the Raman-gain coefficient by using the Kramers-Kronig relation to translate from the imaginary part to the real part and multiplying by $c / 4 \omega$.

It has been reported that the intensity-dependent refractive index increases with the germanium content [37], and that $20 \%$ of the nonlinear refractive index is caused by nuclear motion [31]. At the same time, it is also known that the Raman cross section is about nine times higher in $\mathrm{GeO}_{2}$ relative to $\mathrm{SiO}_{2}$ [26]. Thus, it is interesting to evaluate the Raman contribution to the nonlinear refractive index for $\mathrm{GeO}_{2}$ as well as for $\mathrm{SiO}_{2}$.

In Fig. 5, the Raman contribution from $\mathrm{GeO}_{2}$ to the nonlinear refractive index is displayed together with the similar contribution from $\mathrm{SiO}_{2}$. The figure shows that the Raman contribution to the nonlinear refractive index is about 3.5 times higher in $\mathrm{GeO}_{2}$ than it is in $\mathrm{SiO}_{2}$.

Nuclear motions are not only related to Raman scattering, i.e., phonons at optical frequencies, but also to Brillouin scattering, i.e., phonons at acoustic frequencies. As opposed to the Raman-gain spectrum, the Brillouin spectrum is very narrow (full-width at half-maximum); $\Delta \nu_{\mathrm{B}} \approx 17 \mathrm{MHz}$ for a pump wavelength around $1525 \mathrm{~nm}$ [32]. In addition, the Brillouingain spectrum is centered $\approx 10.5 \mathrm{GHz}$ from the pump where the Raman peak occurs at $13 \mathrm{THz}$ from the pump. Evaluating the contribution to the nonlinear refractive index from Brillouin scattering, as done for the Raman contribution, it is found that the Brillouin contribution is insignificant, because of the narrow Brillouin-gain spectrum.

Since the nonlinear refractive index is known for both $\mathrm{SiO}_{2}$ and $\mathrm{GeO}_{2}$, the fractional contribution to $n_{2}$ from Raman scattering may then be evaluated using Fig. 5. The results of this are shown in Table II ( $f_{r}$ is the nuclear contribution to intensitydependent refractive index).

From Table II, it is noted that even though $n_{2}$ is 4.5 times higher in $\mathrm{GeO}_{2}$ than it is in $\mathrm{SiO}_{2}$, the Raman contribution to $n_{2}$ 
TABLE II

$n_{2}$ VAlues From [35]. For COMPARison, a VAlue of 0.18 For $f_{r}$ IS Noted In [31], Whereas IN [34], A VAlue of 0.3 FOR $f_{r}$ IS QUOTED

\begin{tabular}{|l|c|c|c|}
\hline Material & $n_{2}\left[10^{-20} \mathrm{~m}^{2} / W\right]$ & $n_{2}^{R}\left[10^{-20} \mathrm{~m}^{2} / W\right]$ & $f_{r}$ \\
\hline \hline $\mathrm{GeO}_{2}$ & 12.5 & 1.6 & 0.13 \\
\hline $\mathrm{SiO}_{2}$ & 2.76 & 0.6 & 0.22 \\
\hline
\end{tabular}

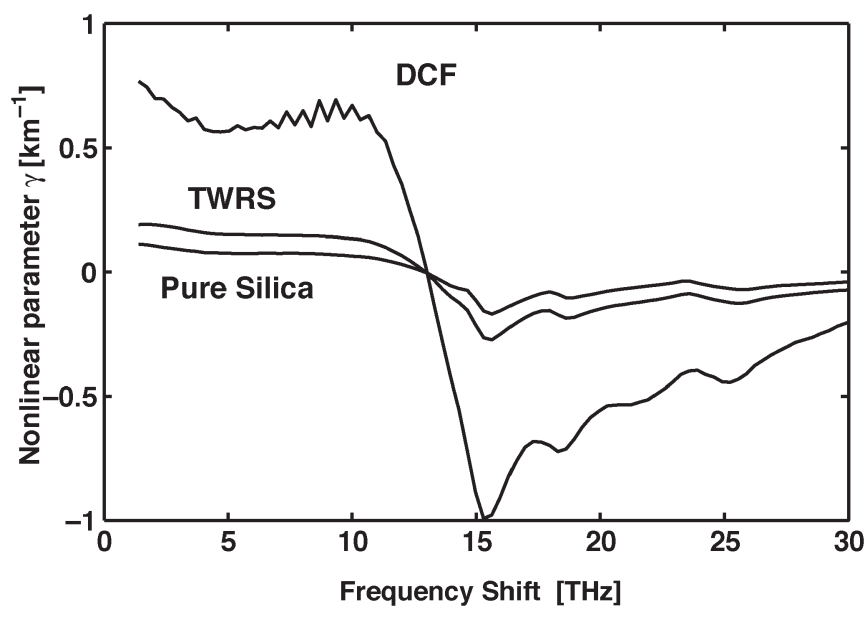

Fig. 6. Raman-induced contribution to the nonlinear parameter $\gamma$, defined in the main text, as a function of the frequency shift between pump and signal.

is only 2.7 times higher for $\mathrm{GeO}_{2}$ as compared to that for $\mathrm{SiO}_{2}$. This implies that the electronic contribution for $\mathrm{GeO}_{2}$ is significantly higher for $\mathrm{GeO}_{2}$ as compared to that for $\mathrm{SiO}_{2}$.

In an optical fiber, the situation is more complicated since the effective area is equally as important as the material parameter. When describing pulse propagation, it makes more sense to evaluate the nonlinear parameter $\gamma$ defined as

$$
\gamma=\frac{2 \pi}{\lambda} \frac{n_{2}}{A_{\mathrm{eff}}}
$$

Fig. 6 displays the Raman contribution to the nonlinear parameter for the three fiber types illustrated in Fig. 1. For comparison, $\gamma$ is typically in the range 1 to $10(\mathrm{~W} \cdot \mathrm{km})^{-1}$ depending on fiber type [38], [39].

\section{CONCLUSiON}

The Raman fiber amplifier has attracted much attention since the mid-1990s. This is explained by its capability to provide distributed amplification, and hence improved noise performance, in addition to gain at any wavelength, and finally, the gain bandwidth may be expanded simply by using multiple pump wavelengths. In addition to these benefits, the Raman scattering is intrinsic to any silica-based fiber.

In this paper, we have reviewed the basic properties of the Raman fiber amplifier. In addition, we have demonstrated the impact of the fiber-design parameters on the Raman-gain coefficient; this includes the germanium concentration, as well as the waveguide design.

Besides essentially being an important parameter for the Raman-amplifier performance, the Raman-gain coefficient may also provide information regarding the response function of the Raman process as well as the contribution to the nonlinear refractive index. We have demonstrated the difference in response time and in contribution to the nonlinear refractive index, comparing silica versus germanate glass.

\section{REFERENCES}

[1] R. H. Stolen and E. P. Ippen, "Raman gain in glass optical waveguides," Appl. Phys. Lett., vol. 22, no. 6, pp. 276-281, 1973.

[2] L. F. Mollenauer, R. H. Stolen, and M. N. Islam, "Experimental demonstration of soliton propagation in long fibers: Loss compensated by Raman gain," Opt. Lett., vol. 10, no. 5, p. 229, May 1985.

[3] P. B. Hansen, L. Eskildsen, S. G. Grubb, S. K. Korotky, T. A. Strasser, J. E. J. Alphonsus, J. J. Veselka, D. J. DiGiovanni, D. W. Peckham, and D. Truxal, "442 km repeaterless transmission in a $10 \mathrm{~Gb} / \mathrm{s}$ system experiment," Electron. Lett., vol 32, no. 11, pp. 1018-1019, May 1996.

[4] T. N. Nielsen, A. J. Stentz, K. Rottwitt, D. S. Vengsarkar, Z. J. Chen, P. B. Hansen, J. H. Park, K. S. Feder, S. Cabot, S. Stulz, D. W. Peckham, L. Hsu, C. K. Kan, A. F. Judy, S. Y. Park, L. E. Nelson, and L. Gruner-Nielsen, "3.28-Tb/s transmission over $3 \times 100 \mathrm{~km}$ of nonzerodispersion fiber using dual C- and L-band distributed Raman amplification," IEEE Photon. Technol. Lett., vol. 12, no. 8, pp. 1079-1081, Aug. 2000.

[5] B. Chu, L. Leng, L. E. Nelson, L. Gruner-Nielsen, Y. Qian, J. Bromage, S. Stultz, S. Kado, Y. Emori, S. Namiki, P. Gaarde, A. Judy, B. Palsdottir, and R. L. Lingle, Jr., "3.2 Tb/s $(80 \times 42.7 \mathrm{~Gb} / \mathrm{s})$ transmission over $20 \times 100 \mathrm{~km}$ of non-zero dispersion fiber with simultaneous $\mathrm{C}+\mathrm{L}$ band dispersion compensation," Proc. Optical Fiber Communication (OFC), Anaheim, CA, 2002, pp. FC8-1-FC8-3, Post deadline paper FC8.

[6] M. Nissov, C. R. Davidson, K. Rottwitt, R. Menges, P. C. Corbett, D. Innis, and N. S. Bergano, " $100 \mathrm{~Gb} / \mathrm{s}(10 \times 10 \mathrm{~Gb} / \mathrm{s})$ WDM transmission over $7200 \mathrm{~km}$ using distributed Raman amplification," in Eur. Conf. Optical Communication (ECOC), Edinburgh, U.K., 1997, pp. 9-12, pdp.

[7] B. Mikkelsen, G. Raybon, R.-J. Essiambre, A. J. Stentz, T. N. Nielsen, D. W. Peckham, L. Hsu, L. Gruner-Nielsen, K. Dreyer, and J. E. Johnson, "320-Gb/s single-channel pseudolinear transmission over $200 \mathrm{~km}$ of nonzero-dispersion fiber," IEEE Photon. Technol. Lett., vol. 12, no. 10 , p. 1400 , Oct. 2000.

[8] C. Xu, X. Liu, L. Mollenauer, and X. Wei, "Comparison of return-tozero differential phase-shift keying and ON-OFF keying long haul dispersion managed transmission," IEEE Photon. Technol. Lett., vol. 15, no. 4, p. 617, Apr. 2003.

[9] S. Wabnitz and G. L. Meur, "Nonlinear and noise limitations in dispersion-managed soliton wavelength-division multiplexing transmission with distributed Raman amplification," Opt. Lett., vol. 26, no. 11, p. 777 , Jun. 2001

[10] E. Desurvirre, Erbium Doped Fiber Amplifiers, Principles and Applications. San Diego, CA: Academic, 1995

[11] C. Lester, K. Rottwitt, J. H. Povlsen, P. Varming, M. Newhouse, and A. J. Antos, "91 km attenuation-free transmission with low noise accumulation by use of distributed erbium-doped fiber," Opt. Lett., vol. 20, no. 11 , pp. 1250-1252, Jun. 1995.

[12] J. Bromage, "Raman amplification for fiber communications systems," J. Lightw. Technol., vol. 22, no. 1, pp. 79-93, Jan. 2004.

[13] H. Kidorf, K. Rottwitt, M. Nissov, M. Ma, and E. Rabarijaona, "Pump interactions in a 100-nm bandwidth Raman amplifier," IEEE Photon. Technol. Lett., vol. 11, no. 5, pp. 530-532, May 1999.

[14] K. M. Hillingsøe, T. V. Andersen, H. N. Paulsen, C. K. Nielsen, K. Mølmer, S. Keiding, R. Kristiansen, K. P. Hansen, and J. J. Larsen, "Supercontinuum generation in a photonic crystal fiber with two zero dispersion wavelengths," Opt. Express, vol. 12, no. 6, pp. 1045-1054, Mar. 2004.

[15] S. N. Knudsen, B. Zhu, L. E. Nelson, M. Ø. Pedersen, D. W. Peckham, and S. Stulz, " $420 \mathrm{~Gb} / \mathrm{s}(42 \times 10 \mathrm{~Gb} / \mathrm{s})$ WDM transmission over $4000 \mathrm{~km}$ of ultrawave fiber with $100 \mathrm{~km}$ dispersion-managed spans and distributed Raman amplification," Electron. Lett., vol. 37, no. 15, p. 965, Jul. 2001.

[16] R. Hellwarth, "Third-order optical susceptibilities of liquids and solids," Prog. Quantum Electron., vol. 5, pt. 1, pp. 2-68, 1977.

[17] R. Hellwarth, J. Cherlow, and T. Yang, "Origin and frequency dependence of nonlinear optical susceptibilities of glasses," Phys. Rev. B, vol. 11, no. 2, pp. 964-967, Jan. 1975. 
[18] D. J. Dougherty, F. X. Kartner, H. A. Haus, and E. P. Ippen, "Measurement of the Raman gain spectrum of optical fibers," Opt. Lett., vol. 20, no. 1, p. 31, Jan. 1995.

[19] S. Seikai, T. Nakashima, and N. Shibat, "Theory of signal light amplification by stimulated Raman scattering in twisted single-mode optical fibers," J. Lightw. Technol., vol. LT-4, no. 6, pp. 583-589, Jun. 1986.

[20] P. B. Hansen, V. L. da Silva, G. Nykolak, J. R. Simpson, D. L. Wilson, J. E. J. Alphonsus, and D. J. DiGiovanni, "374-km transmission in a $2.5-\mathrm{Gb} / \mathrm{s}$ repeaterless system employing a remotely pumped erbiumdoped fiber amplifier," IEEE Photon. Technol. Lett., vol. 7, no. 5, pp. 588-590, May 1995.

[21] E. Desurvire, M. J. F. Digonnet, and H. J. Shaw, "Theory and implementation of a Raman active fiber delay line," J. Lightw. Technol., vol. LT4, no. 4, pp. 426-443, Apr. 1986.

[22] K. Rottwitt, J. Bromage, A. J. Stentz, L. Leng, M. E. Lines, and H. Smith, "Scaling of the Raman gain coefficient: Applications to germanosilicate fibers," J. Lightw. Technol., vol. 21, no. 7, p. 1652, Jul. 2003.

[23] K. Rottwitt and A. J. Stentz, "Raman amplification in lightwave communication systems," in Proc. Optical Fiber Telecommunications (OFC), vol. IVA. San Diego, CA: Academic, 2000, ch. 5, Improved noise perf.

[24] J. Bromage, K. Rottwitt, and M. E. Lines, "A method to predict the Raman gain spectra of germanosilicate fibers with arbitrary index profiles," IEEE Photon. Technol. Lett., vol. 14, no. 1, pp. 24-26, Jan. 2002.

[25] M. N. Islam, Raman Amplifiers for Telecommunications, 1 \& 2. New York: Springer-Verlag, Nov. 2003.

[26] F. L. Galeener, J. C. Mikkelsen, Jr., R. H. Geils, and W. J. Mosby, "The relative Raman cross sections of vitreous $\mathrm{SiO}_{2}, \mathrm{GeO}_{2}, \mathrm{~B}_{2} \mathrm{O}_{3}$, and $\mathrm{P}_{2} \mathrm{O}_{5}$," Appl. Phys. Lett., vol. 32, no. 1, pp. 34-36, Jan. 1978.

[27] M. E. Lines, "Raman-gain estimates for high-gain optical fibers," J. Appl. Phys., vol. 62, no. 11, pp. 4363-4370, Dec. 1987.

[28] Q. Lin and G. P. Agrawal, "Statistics of polarization-dependent gain in fiber-based Raman amplifiers," Opt. Lett., vol. 28, no. 4, pp. 227-229, Feb. 2003.

[29] M. E. Fermann, "Single-mode excitation of multimode fibers with ultrashort pulses," Opt. Lett., vol. 23, no. 1, pp. 52-54, Jan. 1998.

[30] M. J. Adams, An Introduction to Optical Waveguides. New York: Wiley, 1981.
[31] R. Stolen, J. P. Gordon, W. J. Tomlinson, and H. A. Haus, "Raman response function of silica-core fibers," J. Opt. Soc. Amer. B, vol. 6, no. 6 , p. 1159 , Jun. 1989

[32] G. P. Agrawal, Nonlinear Fiber Optics. San Diego, CA: Academic, 1995.

[33] S. Kumar, A. Selvarajan, and G. V. Anand, "Influence of Raman scattering on the cross phase modulation in optical fibers," Opt. Commun., vol. 102 , no. 3-4, pp. 329-335, Oct. 1993.

[34] K. J. Blow and D. Wood, "Theoretical description of transient stimulated Raman scattering in optical fibers," IEEE J. Quantum Electron., vol. 25 , no. 12, pp. 2665-2673, Dec. 1989.

[35] D. Hollenbeck and C. D. Cantrell, "Multiple-vibrational-mode model for fiber-optic Raman gain spectrum and response function," J. Opt. Soc. Amer. B, vol. 19, no. 12, pp. 2886-2892, Dec. 2002.

[36] G. E. Walrafen and P. N. Krishnan, "Model analysis of the Raman spectrum from fused silica optical fibers," Appl. Opt., vol. 21, no. 3, pp. 350-359, Feb. 1982.

[37] T. Kato, Y. Suetsugu, and M. Nishimura, "Estimation of nonlinear refractive index in various silica-based glasses for optical fibers," Opt. Lett., vol. 20, no. 22, pp. 2279-2281, Nov. 1995.

[38] K. S. Kim, R. H. Stolen, W. A. Reed, and K. W. Quoi, "Measurement of the nonlinear index of silica-core and dispersion-shifted fibers," Opt. Lett., vol. 19, no. 4, pp. 25-259, Feb. 1994.

[39] A. Boskovic, S. V. Chernikov, J. R. Taylor, L. Gruner-Nielsen, and O. A. Levring, "Direct continuous-wave measurement of $n_{2}$ in various types of telecommunication fiber at $1.55 \mu \mathrm{m}$," Opt. Lett., vol. 21, no. 24 , pp. 1966-1968, Dec. 1996.

Karsten Rottwitt, photograph and biography not available at the time of publication.

Jørn H. Povlsen, photograph and biography not available at the time of publication. 